\title{
Design and Implementation of PDA-based Image Surveillance System for Harbor Security using IP Camera
}

\author{
† Joon-Hwan Shim* \\ * Division of Computer, Control and Electronic Communications Engineering, Korea Maritime University, Busan 606-791, Republic. \\ of Korea
}

\begin{abstract}
This paper describes a new progressive embedded Internet Protocol(IP) camera available for real-time image transmission. It was applied for ship safety and security on seashore area. The functions of developed embedded system was more effective and excellent than conventional systems. Nowadays, each nation has established harbor security systems to jump up their ports to international port. Recently Incheon port has tried to change man security into center security system used by image security system. The security system of Incheon port has some advantages like effectivity of security system and reduction of manpower and cost, installed by image security system with CCTV cameras at the entrance gate and around the fence. Thus in this paper, we have designed and implemented a Personal Digital Assistants(PDA) based Image Surveillance System for Harbor Security using IP Camera under ubiquitous environment. This system has big advantages which are more effective in an emergency and low cost and small manpower than conventional systems.
\end{abstract}

Key words : IP camera, Progressive camera, PDA, Image surveillance system, Harbor security, MPEG-4

\section{Introduction}

After World Trade Center's terror on September 11, 2001 in America and suffering attack of ultra-large oil tanker ship, M/T Limburg of france, security council of each port have tried to enhance the safety and security for freight transport and logistics because there is greater opportunity for terrorists to conceal their attack materials within commercial cargo and containers(Yoon, 2007). Under intensive competition environment among ports, efficient port security system is also required to become a logistics hub of northeast asia in the 21st century.

Particularly, in order to prepare for the possibility of terrorism against port facilities and marine transport like container ship, LPG vessel, LNG vessel, International Code for the Security of Ships and Port Facility(ISPS Code) (IMO, 2003), which was developed by Maritime Safety Committee(MSC) of International Maritime Organization (IMO), was adopted in 2001 and entered into force on July 1, 2004. According to the regulation, it is a purpose to detect and deter terrorisms against port facilities by issuing suitability certificate of port facility about every port facilities capable of accommodating cargo ships of 500 tons and above.

Nowadays, departments of harbor security of each nation have tried to implement efficient harbor security system to cope with new maritime security measures(McDonald, 2004;
Park, 2005). Recently Incheon port has tried to change man-controlled security system into new center-controlled security system using real-time image security system. The security system of Incheon port has some advantages like high effectivity of security system and reduction of manpower and cost, installed by image security system with CCTV cameras at the entrance gate and around the fence.

In this paper, we have designed and implemented PDA-based image surveillance system using IP camera. Because IP camera can be used by broadband wireless LAN communication without PC server, they have some merits of being capable of monitoring real-time images of IP camera by mobile phone and PDA at access point available with internet connection all over the world. Owing to these reasons, many researchers have studied extensively industrial applications using IP camera(Hochmuth, 2005; Zhao, 2006; Cager, 2006 ; Kang, 2007).

The proposed system also has great advantages of manpower cost reduction and big efficiency of emergency operations compared to the conventional security system because it is possible for officers far away from the main control center to monitor image information of harbor at any time and any place. And this system is capable of effectively transmitting real-time image through CDMA and satellite communication because it adopted progressive scan method available for clear image data transfer and high

† Corresponding Author : Joon-Hwan Shim, jhsim@.hhu.ac.kr 051)410-4811 
MPEG-4 image compression transfer technique.

\section{Design of image security system}

\subsection{System architecture overview}

Fig. 1 shows an architecture of real-time image surveillance monitoring system for harbor facilities suggested in this paper.



Fig. 1 Architecture of real-time image surveillance monitoring system.

As shown in Fig. 1, firstly, real-time images in main surveillance regions of port are detected by the progressive IP cameras and secondly, the images are transformed into MPEG-4 compressive images from IP camera modules, and then the compressed images are transmitted through the network.

Next, the transmitted images are stored in the data server and the information can be observed in the main control center connected to the server.

Also, when a specific IP address is sent into the network by a security officer, the fixed IP camera with the IP address is then connected to the terminal. Thus images of IP camera are compressed by MPEG- 4 method and the images are displayed on PDA or PC terminal

Therefore, this system can increase efficiency for emergency management because, at any time and any place, any officer far away from the main control center is able to access image information of harbor with PDA.

\subsection{Hardware system structure}

The developed progressive embedded real-time IP camera through the network image transmission in this research can effectively transmit by adopting the progressive scan method available for clear image date transfer and high
MPEG-4 image compression transfer technique.

As at transmitting images with at least network speed (144kbs), this system can transmit real-time images with high frame(7f/sec or over) utilized by MPEG-4 compression technique and exactly analyze the captured images, which have high quality in video by the progressive scan technique.

Thus the proposed system has superior performance compared to the conventional image transmission method by a mobile phone, which transmit still images with a transmission speed(1f/sec, JPEG format).

Fig. 2 shows an architecture of embedded system of IP camera module for transmitting real-time image and voice. This system can utilize real-time MPEG- 4 compression of moving image and various protocols such as TCP/IP, UDP/IP, FTP, HTTP, etc. The image signal is read out from the CCD image sensor. And it is capable of monitoring image through WIPI-based mobile phones and includes multi-codec for real-time networking service.

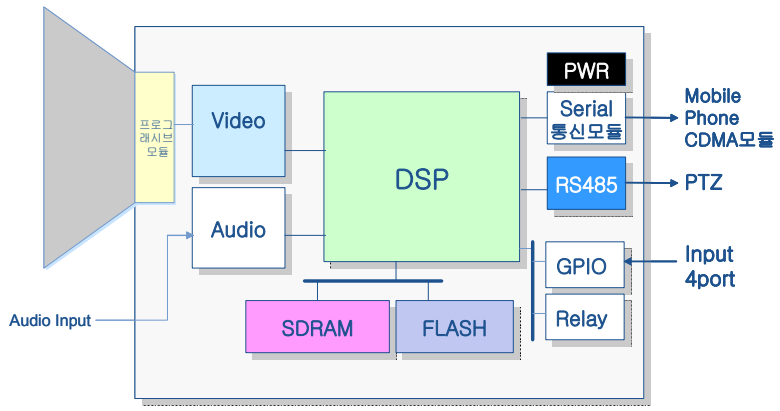

Fig. 2 Architecture of embedded system of IP camera

Table 1 shows specifications of the developed embedded system for real-time image transmission. Ten users can simultaneously connect in this system and it can be extended up to 100 users if needed. And basic internal memory is 16Mbytes SDRAM but it also can be extended.

Though the MPEG-1, 2 have sought for high-quality image, they don't have efficient compression rate(Li, 2001(a) ; Li, 2001(b) ; Sugano, 2001). However this system can obtain high-quality video with low bit-rate image compression. To fit this purpose, many functions were integrated onto one chip without any additional operating software using MPEG-4 compression codec. In the case of the conventional system using MPEG-4 system, it is necessary to use different four chips for voice, network, OS, MPEG-4 system(Chang, 2003). Thus the system still appears to be having some problems about compatibility between each chip and degrade reliability and safety.

But in this research, every function of the suggested system was integrated onto one chip with voice, network, 
OS, MPEG-4 chip and thus the system is excellent in the way of reliability and safety. Fig. 3 shows a web video board fabricated using DSP chip, which integrates the functionality of four chips into one.

Table 1 Spec. of the developed embedded system.

\begin{tabular}{|l|l|}
\hline Standards & MPEG-4 advanced simple profile \\
\hline Video Resolution & QVGA (320x240)/HVGA (640X240) \\
\hline Encoding Frame Rates & 1ch QVGA: max. 60 fps \\
\hline Video Inputs & $\begin{array}{l}\text { 1ch. input, } \\
\text { NTSC/PAL 75 } \Omega, \text { 1Vpp }\end{array}$ \\
\hline Audio Encoding & $\begin{array}{l}\text { G.726, ADPCM 8KHz sampling, } \\
\text { G.723.1 CELP 8KHz sampling, } \\
\text { 6.3Kbps (optional) }\end{array}$ \\
\hline Bi-Directional Audio & $\begin{array}{l}\text { Microphone input: 1 channel } \\
\text { Loudspeaker output: 1 channel }\end{array}$ \\
\hline Protocols & $\begin{array}{l}\text { TCP/IP, UDP/IP, HTTP, DHCP, } \\
\text { ARP, PING, FTP }\end{array}$ \\
\hline LAN Ports & 10 BASE-T, RJ-45, 1 ea \\
\hline SIO & $\begin{array}{l}\text { RS232 } \\
\text { RS485/422(optional) }\end{array}$ \\
\hline OS Support & Windows 98, 2000, ME, XP \\
\hline LED Display & Power, Link, LAN \\
\hline Power & DC 12V, 500 mA \\
\hline GPIO & $\begin{array}{l}\text { TTL level inputs: 2 } \\
\text { TTL level outputs: 3 }\end{array}$ \\
\hline
\end{tabular}

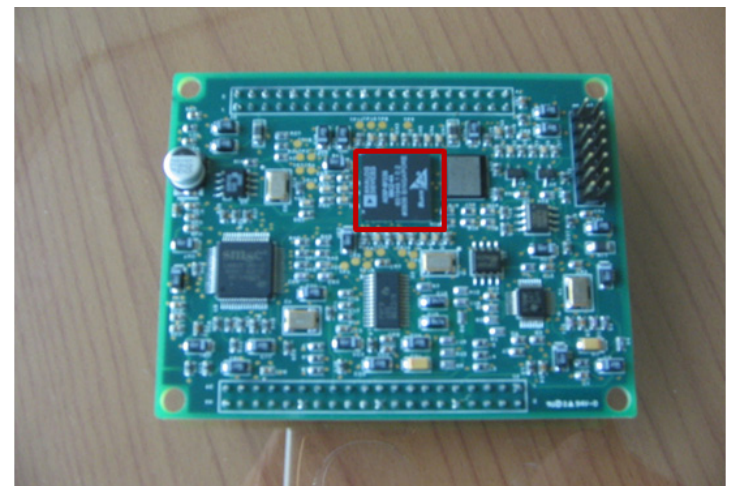

Fig. 3 Picture of web video board with fusion DSP.

Because the network transmission speed is assigned a specific value in the case of single codec, if a different network transmission speed is connected, the system is displayed at low frame rates. But as shown in Fig. 4, owing to the applied multi-codec in this system, it can provide image service according to user's environments, which have different image compression according to user's network transmission speed as it is divided into four different bandwidth.



Fig. 4 Comparison of single and multi codec.

The methods for transmitting image are interlace scan and progressive scan as shown in Fig. 5. The interlace scan is the method that odd-line image is first sent and then even-line image is sent but the progressive scan is the method that each image is alternately sent in sequence(Van de Ville, 2000; Hur, 2001). The conventional progressive method have usually been applied in high-price IP camera system but in this research, as optimizing compression codec, the low-cost high-performance image system was implemented by appling the progressive method to low-price IP camera. The applied progressive method is superior in the way of clearness at still image as shown in Fig. 5(a), compared to the interlace method. And we can recognize that the progressive method is also more clearer at moving image as shown in Fig. 5(b) and thus if the system is applied to a surveillance system, the image will be able to be utilized as an important proof material. Table 2 shows the primary difference between the developed embedded system and other system for real-time image transmission.
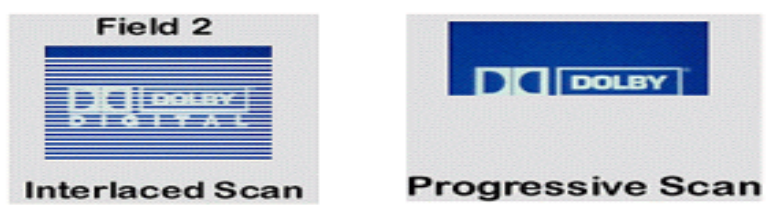

(a) still image

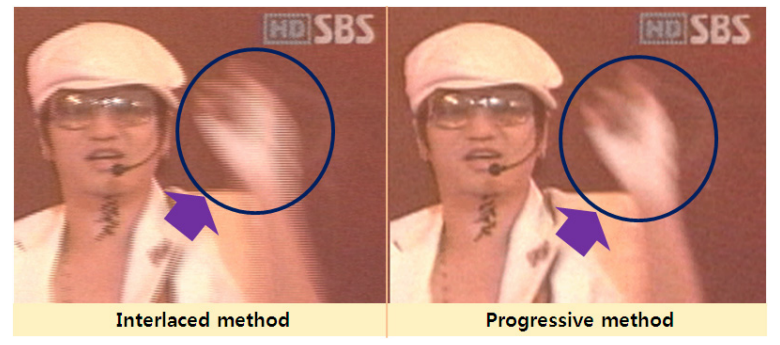

(b) moving image

Fig. 5 Image comparisons between interlaced scan and progressive scan method. 
Table 2 The primary difference between the developed embedded system and other system.

\begin{tabular}{|c|c|c|c|}
\hline & \multicolumn{2}{|c|}{ DSP chip Product } & MPEG-4 chip \\
\cline { 2 - 3 } & $\begin{array}{c}\text { The developed } \\
\text { system }\end{array}$ & Other system & Mroduct \\
\hline $\begin{array}{c}\text { Network } \\
\text { Transmission } \\
\text { Rate }\end{array}$ & Max 60fr/sec & Max 25fr/sec & Max 25fr/sec \\
\hline $\begin{array}{c}\text { Network } \\
\text { transmission } \\
\text { method }\end{array}$ & Multi-codec & Single-codec & Single-codec \\
\hline Board size(cm) & 10 & 15 & $>15$ \\
\hline WMP support & Supported & Not supported & Not supported \\
\hline $\begin{array}{c}\text { Mobile support } \\
\text { Cost(won) } \\
\text { (based on our } \\
\text { system) }\end{array}$ & Total support & PDA support & PDA support \\
\hline
\end{tabular}

\section{Implement of image security system}

Fig. 6 shows the connection results using admin tool, which initiates in network environment for obtaining image information through IP camera. User IP management is the basic tasks of network administrators and they should register, delete and modify the existing users and groups in main system if needed. To do this purpose, the admin tool was used in this administration system.

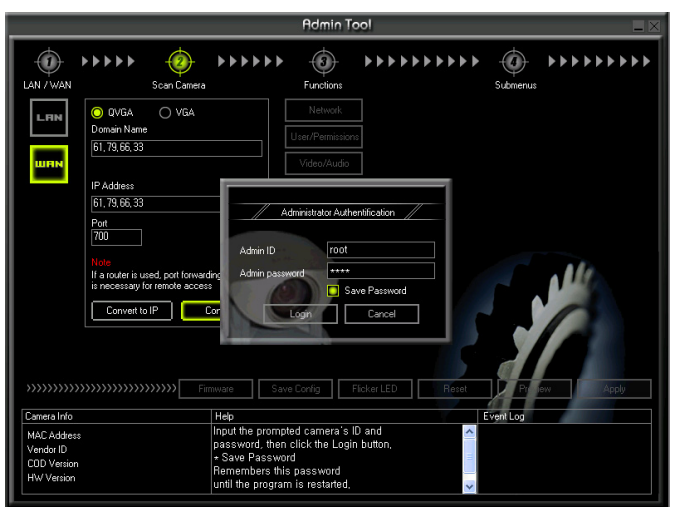

(a) admin tool login

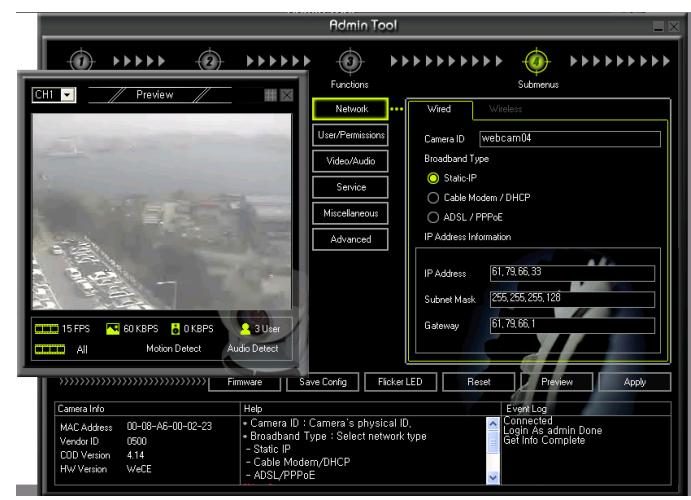

(b) admin tool networking

Fig. 6 Network connection by admin tool.
Admin tool is a tool to be able to administer a data server, which store image information of IP camera through a web browser. It is capable of conforming current states of the server and controling each parameter such as brightness, color, contrast, bit rate of image information.

Fig. 7 shows 4 -channel viewer program of image surveillance system. As a network environment is established by admin tool, this viewer is the image monitoring program to be capable of simultaneously monitoring real-time images of IP cameras, connected to port facilities from main control center, through four channels. This viewer can save images of each channel into data server during the wanted period and because it provides functions to be able to capture images before and after accidence at emergency, security officers can take the necessary measures, comparing with a situation before and after accidence.

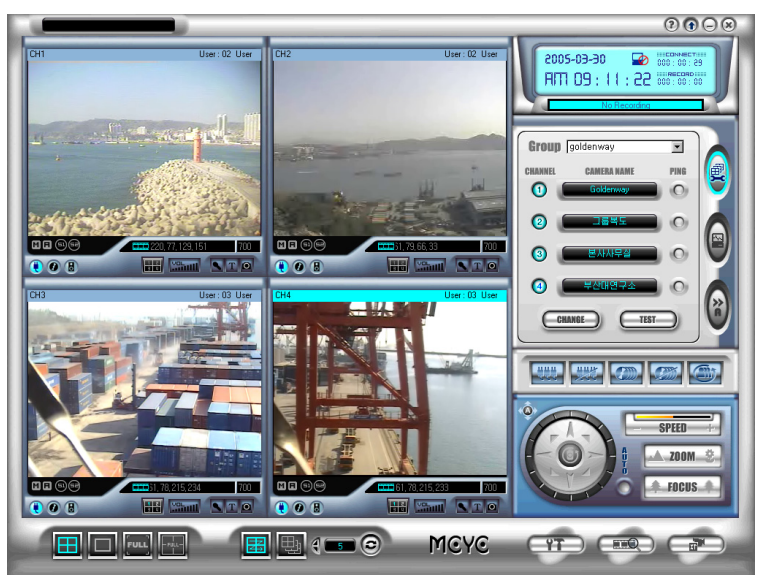

Fig. 74 -channel viewer of image surveillance system.

Fig. 8 shows monitoring process of real-time image of IP camera by PDA using Code Division Multiple Access (CDMA) mobile communication network. To obtain moving image service, user should connect to the given wireless internet server using user's PDA terminal. Wireless internet server can be connected by using Wireless Application Protocol (WAP) and the connected pages is consisted of optimized compression images to reduce transmission load of information. In general, MPEG-4 video standard supports wide range of low bit rate of less 20kbps up to high bit rate of 20Mbps. The developed real-time video encoder is capable of encoding in all the range suggested by standard. As stable transmission speed in CDMA communication network is over 512kbps, the proposed encoder server provide the compressed moving image of above 7 frames per second at the speed of $144 \mathrm{kbps}$ and then one frame become a size of QVGA. 
Joon-Hwan Shim

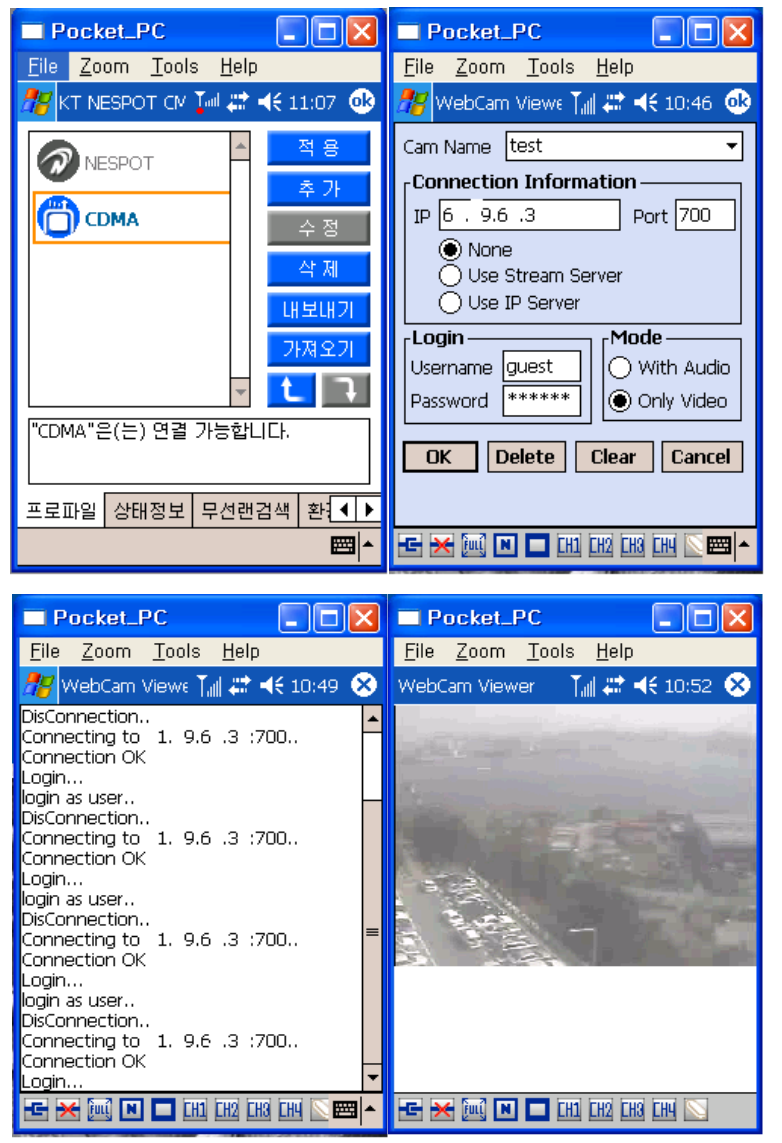

Fig. 8 Image monitoring process of PDA using CDMA mobile communication network.

Fig. 9 shows monitoring process of real-time image of IP camera by PDA using NESPOT connection in wireless internet zone, in which wireless LAN environment is established. As shown in Fig. 10, it shows the experiment results in the test-bed of image surveillance system. It displayed the training ship of Korea Maritime University in harbor by PC terminal with network connection and PDA with CDMA communication connection.

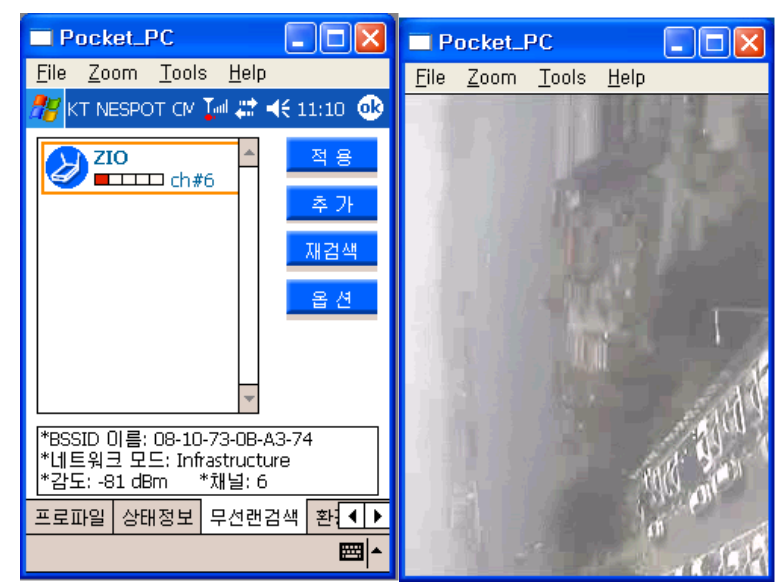

Fig. 9 Image monitoring process of PDA using NESPOT connection in wireless internet zone.

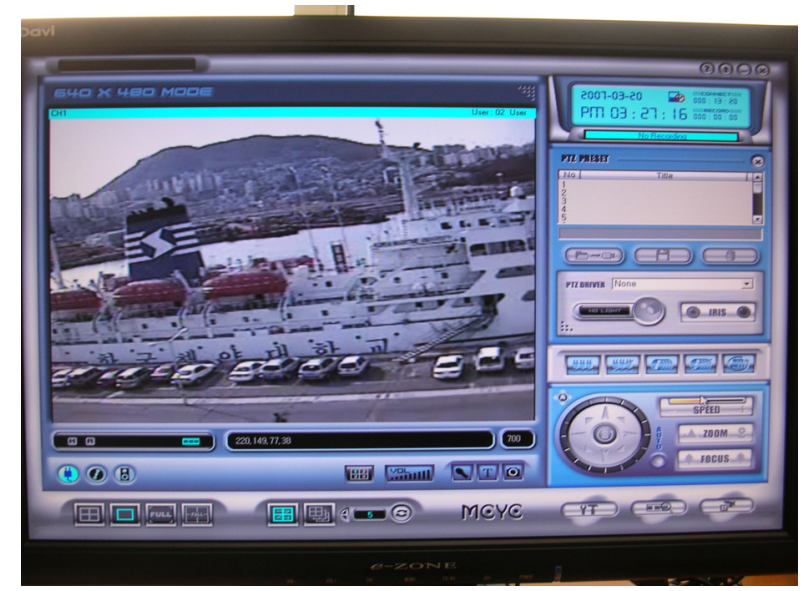

(a) display of PC terminal



(b) display of PDA

Fig. 10 Image monitoring results of $\mathrm{PC}$ and PDA in training ship port of Korea Maritime University.

The current security system of each port have mainly adopted CCTV cameras for image monitoring but in this research, we have designed and implemented PDA-based image surveillance system using IP camera for harbor security. Because IP camera can be used by broadband wireless LAN communication without PC server, they have some advantages of being capable of monitoring real-time images of IP camera by mobile phone and PDA at access point available with internet connection all over the world.

\section{Conclusion}

In this research, we developed embedded IP camera module to implement surveillance system of port facilities under ubiquitous environment and designed and realized PDA-based Image Surveillance System for Harbor Security using IP Camera module. The purpose of this system was to accomplish high-performance and efficient, convenient surveillance system through convergent service offering 
with high-tech multimedia. Thus the developed system benefited greatly from manpower cost reduction and big efficiency of emergency operations compared to the conventional security system because it is possible for officers far away from the main control center to monitor image information of harbor at any time and any place. As stable transmission speed in CDMA communication network is over $512 \mathrm{kbps}$, the developed encoder server is capable of transmitting high-quality, high-clearness image of above 7 frames per second at the speed of $144 \mathrm{kbps}$ and thus this system can be applied to real-time image transmission technique for many diverse applications of transmission media.

\section{Acknowledgements}

The author would like to thank Chan-young Park in Elcom Company for his help with system set-up and kind support with technical comments.

\section{References}

[1] Cager, Y. (2006), "IP Camera Designers Leverage Flexible, Cost-Effective DSP-based Digital Media SoCs for Quality Networked Video", Texas Instruments, White Paper, pp. $1 \sim 6$.

[2] Chang, Y. C. et al. (2003), "Performance Evaluation of MPEG-4 Visual Error Resilient Tools over a Mobile Channel", IEEE Trans. Consumer Electro., vol.49, no.1, pp. $6 \sim 13$.

[3] Hochmuth, P. (2005), "New York courts find security in IP video", Network world, vol.22, no.31, pp. 1 4.

[4] Hur, B. S., Kang, M. G. (2001) "High definition color interpolation scheme for progressive scan CCD image sensor", IEEE Transactions on Consumer Electronics, vol.47, Issue 1 , pp. $179 \sim 186$.
[5] Yoon, D. G. (2007), "A Sensitivity Risk Analysis for Additional Truck Turnaround Time(ATTT) by Container Inspection Stations Derived from C-TPAT and CSI", Journal of Korean Navigation and Port Research, Vol.31, No.2, pp. 151 157.

[6] IMO (2003), "International Ship and Port Facility Security Code(ISPS)", 2003 Edition.

[7] Kang, M. Y. et al. (2007), "A Live Webcasting System using IP camera", Korea Computer Congress 2007, vol.34, no.1(D), pp. 315 320.

[8] Li, X. M. (2001), "A novel random access approach for MPEG-1 multicast applications", 2001 International Conferences on Info-tech and Info-net (ICII 2001), vol.2, pp. $413 \sim 417$.

[9] Li, S. H., Zhu, H. W. and Li, J. H. (2001), "Study of a new model for MPEG-1 video traffics in ATM networks", 2001 IEEE Third Workshop on Signal Processing Advances in Wireless Communications (SPAWC '01), pp. 391 394.

[10] McDonald, L. and O Sulivan, R. (2004), "Integrated Harbor Security System Enhances Port Protection", Sea Technology, vol.45, no.2, pp. 27 30.

[4] Park, J. (2005), "A study on the practical strategies of maritime security system", Master's Thesis, Segang University.

[11] Sugano, M. et al. (2001), "An efficient transcoding from MPEG-2 to MPEG-1", 2001 International Conference on Image, vol.1, pp. 417 420.

[13] Van de Ville, D. et al. (2000), "Motion compensated de-interlacing for both real time video and still images", 2000 International Conference on Image Processing, vol.2, pp. 680 683.

[14] Zhao, Y. and Taubin, G. (2006) "Real-time median filtering for embedded smart cameras", IEEE International Conference on Computer Vision Systems(ICVS 2006), pp. 55 60.

Received 7 December 2007

Accepted 31 December 2007 